\title{
A novel panel of mouse models to evaluate the role of human pregnane $X$ receptor and constitutive androstane receptor in drug response
}

\author{
Nico Scheer, ${ }^{1}$ Jillian Ross, ${ }^{2}$ Anja Rode, ${ }^{1}$ Branko Zevnik, ${ }^{1}$ \\ Sandra Niehaves, ${ }^{1}$ Nicole Faust, ${ }^{1,3}$ and C. Roland Wolf ${ }^{2,4}$
}

\begin{abstract}
${ }^{1}$ TaconicArtemis, Cologne, Germany. ${ }^{2} \mathrm{CXR}$ Biosciences Ltd., Dundee, United Kingdom. ${ }^{3}$ amaxa AG, Cologne, Germany. ${ }^{4}$ Cancer Research UK Molecular Pharmacology Unit, Biomedical Research Centre, Ninewells Hospital and Medical School, University of Dundee, Dundee, United Kingdom.
\end{abstract}

\begin{abstract}
The pregnane $X$ receptor (PXR) and the constitutive androstane receptor (CAR) are closely related orphan nuclear hormone receptors that play a critical role as xenobiotic sensors in mammals. Both receptors regulate the expression of genes involved in the biotransformation of chemicals in a ligand-dependent manner. As the ligand specificity of PXR and CAR have diverged between species, the prediction of in vivo PXR and CAR interactions with a drug are difficult to extrapolate from animals to humans. We report the development of what we believe are novel PXR-and CAR-humanized mice, generated using a knockin strategy, and Pxr-and Car-KO mice as well as a panel of mice including all possible combinations of these genetic alterations. The expression of human CAR and PXR was in the predicted tissues at physiological levels, and splice variants of both human receptors were expressed. The panel of mice will allow the dissection of the crosstalk between PXR and CAR in the response to different drugs. To demonstrate the utility of this panel of mice, we used the mice to show that the in vivo induction of Cyp3a11 and Cyp2b10 by phenobarbital was only mediated by CAR, although this compound is described as a PXR and CAR activator in vitro. This panel of mouse models is a useful tool to evaluate the roles of CAR and PXR in drug bioavailability, toxicity, and efficacy in humans.
\end{abstract}

\section{Introduction}

Mammals are continuously exposed to chemicals from external sources. To overcome the potentially harmful effects of these xenobiotics, a detoxifying mechanism has evolved that has been categorized into phase I, phase II, and phase III. Phase I largely relies on the biotransformation of xenobiotics by enzymes encoded by the superfamily of cytochrome P450 (CYP) genes $(1,2)$. The phase I metabolites can either be eliminated directly from the body or are subject to further biotransformation by phase II enzymes prior to elimination. The primary organ of drug metabolism is the liver, but other organs such as the intestine or lung can also be involved, as reflected by the expression of CYP enzymes in these organs $(3,4)$. In addition, transporters, sometimes called phase III proteins, can determine foreign compound bioavailability, distribution, and elimination.

The genes involved in drug metabolism provide an adaptive response to environmental challenge, and as a consequence,

Nonstandard abbreviations used: $\mathrm{BQ}, 7$-benzyloxyquinoline; $\mathrm{CAR}$, constitutive androstane receptor; CITCO, 6-(4-chlorophenyl)imidazo-[2,1-b][1,3] thiazole-5-carbaldehydeO-(3,4-dichloro-benzyl)oxime; CYP, cytochrome P450; DEX, dexamethasone; eFLP, efficient FLP; FLP, flipase recombinase; hCAR, human CAR; hPXR, human PXR; huCAR, humanized CAR (mice); huPXR, humanized PXR (mice); huPXR/ huCAR, PXR and CAR double humanized (mice); LBD, ligand-binding domain; mCAR, murine CAR; mPXR, murine PXR; PB, phenobarbital; PCN, pregnenolone$16 \propto$-carbonitrile; $\mathrm{PROD}$, pentoxyresorufin dealkylation; $\mathrm{PXR}$, pregnane $\mathrm{X}$ receptor; qRT-PCR, quantitative RT-PCR; RIF, rifampicin; TCPOBOP, 1,4-bis[2-(3,5-dichlorop yridyloxy)] benzene.

Conflict of interest: N. Scheer, A. Rode, B. Zevnik, and S. Niehaves receive income from TaconicArtemis; J. Ross and C.R. Wolf receive income from CXR Biosciences; and N. Faust receives income from amaxa AG.

Citation for this article: J. Clin. Invest. 118:3228-3239 (2008). doi:10.1172/JCI35483. their expression is often tightly regulated by foreign compounds themselves (5). Recently, it has become clear that the orphan nuclear hormone receptors pregnane $\mathrm{X}$ receptor (PXR) and constitutive androstane receptor (CAR) play a critical role in the transcriptional regulation of many phase I and phase II enzymes and transporters (6).

The general structure of PXR and CAR is well conserved between species, including a highly variable $\mathrm{N}$-terminal domain, a central DNA-binding domain, and a C-terminal ligand-binding domain $(\operatorname{LBD})(7,8)$. Upon ligand binding, the receptors translocate from the cytoplasm to the nucleus, where they regulate the expression of genes with corresponding binding enhancer elements in their promoters $(9,10)$. Both PXR and CAR interact with a variety of different ligands $(6,11)$.

Despite a well-conserved structure for PXR and CAR between species and a high degree of similarity in the DNA-binding domain in particular, the LBD has diverged significantly (11). Both receptors bind to distinct ligands as well as to the same ligand, but with different affinities. These interactions have probably developed as an adaptation to diverse environmental conditions. For example, the synthetic C21 steroid pregnenolone-16 $\propto$-carbonitrile (PCN) is a potent ligand of murine PXR (mPXR) but not human PXR (hPXR), while the macrocyclic antibiotic rifampicin (RIF) is more selective for the human receptor (12). On the other hand, human CAR (hCAR) but not murine CAR (mCAR) is strongly activated by 6-(4-chlorophenyl)imidazo-[2,1-b][1,3]thiazole-5-carbaldehydeO(3,4-dichloro-benzyl)oxime (CITCO) (13), but the mouse receptor is more sensitive to 1,4-bis[2-(3,5-dichloropyridyloxy)]benzene (TCPOBOP) than hCAR $(14,15)$. Other compounds, such as phe- 
nobarbital (PB) have been shown to interact with both PXR and CAR in vitro (16), and the relative contribution of both receptors for the in vivo regulation of downstream genes is unknown.

The critical role of PXR and CAR in the regulation of phase I and II enzymes as well as transporters on the one hand and their selectivity for different ligands in distinct species on the other can give rise to profound differences in drug bioavailability, distribution, toxicity, and efficacy between animals and humans.

To overcome these problems, humanized mouse models for PXR and CAR have been generated, in which the murine receptors have been deleted and the hCAR and hPXR cDNAs under control of the liver-specific albumin promoter $(12,17)$ or the rat fatty acid-binding protein promoter (18) randomly integrated into the mouse genome. However, due to the use of heterologous promoters, these models do not provide bona fide levels of expression of PXR or CAR in all tissues of the mouse. Furthermore, splice variants of hPXR and hCAR have recently been described, some of which have been shown to have functional activity (19-23). None of these splice variants are expressed by mouse models in which pure cDNAs are used. Recently, Ma et al. generated an improved model for PXR in which a genomic fragment containing the entire $h P X R$ gene and its promoter have been randomly integrated on a Pxr-null background (24).

The interactions of chemicals with PXR and CAR are complex because they regulate common genes and a single chemical agent can often interact with both receptors (Figure 1). In order to fully understand the relative importance of the receptors in the efficacy and safety of drugs, it is critical to develop models in which the roles of these receptors can be evaluated separately and in combination.

In order to improve the pattern of expression and information derived from humanized PXR (huPXR) and humanized CAR (huCAR) mice, we report the generation and characterization of new humanized mouse models for these receptors. In contrast to previous approaches, we have used knockin strategies to express hPXR and CAR under control of their corresponding mouse promoters, with an interruption of the expression of the endogenous genes. The low genetic complexity of this system, requiring only 1 genetic alteration for each receptor, allowed us to establish a panel of PXR/CAR mouse models. This panel contains single-humanized and KO models of PXR and CAR and all possible combinations of these alterations, including double-humanized (huPXR/ huCAR) and $\mathrm{KO}$ models for both receptors. Besides showing that the human receptors are expressed in the anticipated organs at levels comparable to those in their murine counterparts and that major human splice isoforms of PXR and CAR are expressed, we demonstrate the species-specific interaction of both receptors with selected compounds. Furthermore, we show that the in vivo induction of Cyp3a11 and Cyp2b10 by PB is only mediated by CAR, though in vitro experiments suggest an interaction of this compound with both PXR and CAR (16). Using an in vivo pharmacokinetics approach to identify species-specific CAR inducers, we demonstrate a difference in the metabolism of Cyp3a11 and Cyp2b10 probe substrates in WT and huCAR mice after treatment with CITCO. Finally, we discuss how the panel can be used to dissect the crosstalk of PXR and CAR and to evaluate their contribution to efficacy and safety of a drug in humans.

\section{Results}

Humanization of mice for PXR and CAR by knockin into the endogenous gene locus. huPXR and huCAR mice were generated by a knockin strategy as depicted in Figure 2, so that the expres- sion of the human receptors is controlled by the corresponding mouse promoters. This strategy simultaneously deletes the endogenous gene function.

In the case of PXR, a chimeric construct consisting of the cDNA of exons 2-4 of hPXR missing the start CTG, genomic sequences of intron 4, exon 5, and intron 5 and again cDNA of exons 6-9 followed by an SV40 poly(A) signal were introduced at the ATG of the $m P x r$. The partial conservation of the exon/intron structure in these animals potentially allows the expression of splice isoforms that code for variants with an altered LBD and that have been identified predominantly in the genomic region of exons 4 to 6 (19).

In the case of CAR, essentially the whole human gene, except the noncoding first exon, was introduced, i.e., from exon 2 and all downstream introns and exons of hCAR. This potentially allows the expression of all previously described splice variants of hCAR (21).

By making use of the $\phi \mathrm{C} 31$ recognition sites attB53 and attP50 in the targeting vectors, KOs for both receptors (referred to as PXR $\mathrm{KO}$ and $\mathrm{CAR} \mathrm{KO}$ ) were generated by crossing the corresponding humanized mice to a $\phi C 31$-deleter strain. In the case of $C A R$, this led to a complete removal of all coding sequences except $107 \mathrm{bp}$ of human exon 2 . The translation start site was removed in the case of PXR, but as mouse exons 1 and 3-9 were still present after $\phi C 31$ mediated deletion, we included a splice acceptor poly $(\mathrm{A})$ signal in our targeting vector, which caused splicing of exon 1 to the splice acceptor and terminated transcription by the poly(A) signal. As all exons coding for functional domains of CAR were deleted in CAR $\mathrm{KO}$ and the expression of PXR was prevented in PXR KO, both represent complete KOs for the corresponding receptors. Though the lack of PXR and CAR antibodies that would allow one to detect the receptors in tissue samples rules out a direct verification of the absence of the proteins, the validation experiments described below confirm the functional KO of PXR and CAR in these mouse lines.

Homozygous humanized and KO mice for PXR or CAR appeared normal, could not be distinguished from WT mice, and had normal survival rates and fertility. H\&E analysis on the livers of all the transgenic mouse lines revealed that the microscopic findings recorded were indicative of normal pathology (data not shown).

Stable expression of $h P X R$ and $h C A R$ in liver and intestine of humanized mice. In order to demonstrate that $D P X R$ but not $M P X R$ and CAR mRNA was expressed in the humanized mice and that $m C$ ar and $m P x r$ mRNA expression was lost in the KOs, qualitative PCR analysis was performed (Figure 3). $m P X R$ mRNA was only expressed in the liver and intestine of WT but not in humanized mice, while the DPXR mRNA was expressed in the humanized animals. Comparable results were obtained for the huCAR mice (Figure 3). Furthermore, mRNA expression in the humanized mice was not only detected in the expected tissues but also at similar levels as in the WT animals. In addition, neither mouse nor human mRNA of the corresponding receptor was detected in PXR and CAR KO mice, respectively (data not shown).

Detection of different human splice isoforms in buPXR and huCAR mice. In order to establish whether splice variants of CAR and PXR were produced, we performed a sequence analysis of different CDNA clones obtained by RT-PCR from livers of huPXR and huCAR mice. Full-length transcripts for both hCAR and hPXR were identified in the transgenic lines. In addition, in the case of huCAR mice, 4 human splice isoforms described previously in the literature were identified $(21,22)$ (Figure 4$)$. These are variants with a 12-bp insertion between exons 6 and 7 (SV3), a 15-bp insertion between exons 7 and 8 (SV2), a complete deletion of exon 7 (SV4), 


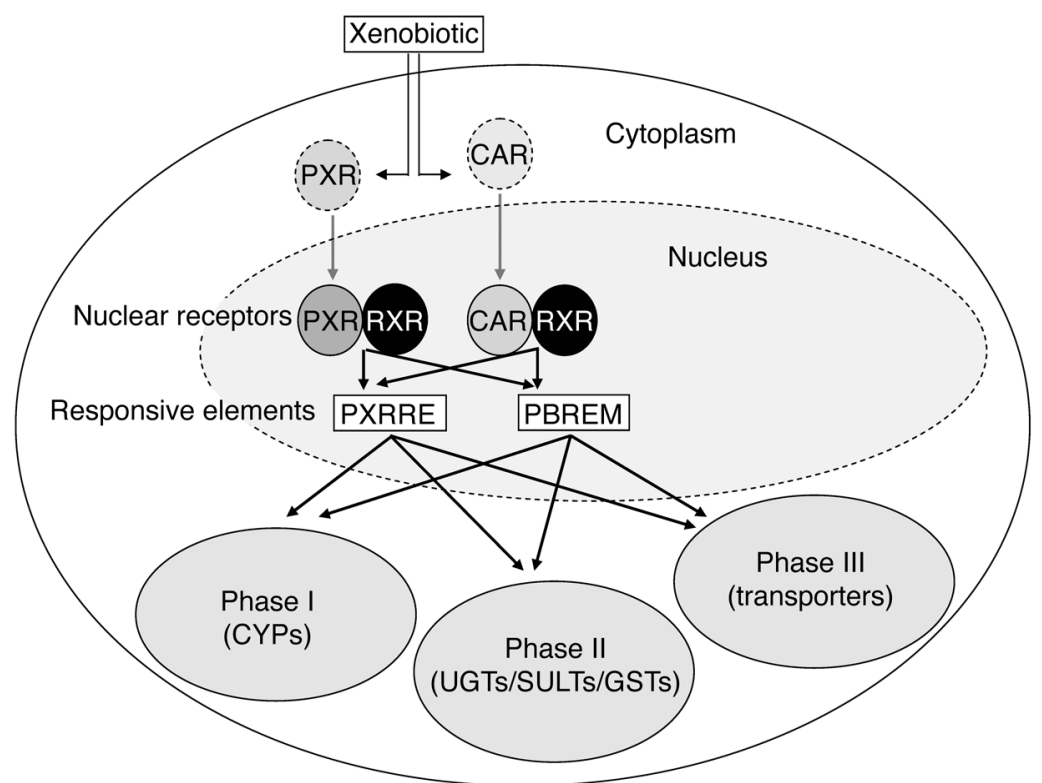

\section{Figure 1}

Crosstalk between PXR and CAR. A multitude of xenobiotics can interact with PXR or CAR or both. Ligand binding leads to the translocation of the receptor from the cytoplasm to the nucleus, where both PXR and CAR use the retinoic $X$ receptor $(R X R)$ as a partner for heterodimerization and bind to corresponding cisacting elements of their target genes. PXR and CAR mediate gene regulation through certain direct repeats, known as the PXR responsive element (PXRRE) and the PB responsive element module (PBREM). Target genes that contain PXRREs and PBREMs in their promoters can be regulated both by PXR and CAR. Among those that are subject to PXR and CAR regulation are a number of genes encoding phase I or phase II drug-metabolizing enzymes or transporters. UGT, uridine diphosphate-glucuronosyltransferase; SULT, sulfotransferase; GST, glutathione S-transferase. and the 12-bp insertion of SV3 together with an out-of-frame deletion of the first 76 bps of exon 9 (SV9). For PXR, the WT and the most abundant splice variant formed in hPXR SV2 (or hPXR.2), coding for a truncated protein lacking 37 amino acids of the LBD $(19,25,26)$ was identified.

In both huPXR and huCAR mice, further and yet unidentified splice variants presumably coding for nonfunctional receptors were detected at low abundance (data not shown).

Functional analysis of the mouse models by using PXR activators. hPXR and CAR exhibit different ligand specificities to the mouse receptors $(6,14)$. In order to establish functional activity of PXR and CAR proteins and the phenotype of the animals, WT, humanized, and null mice were treated with compounds known to induce gene expression through these transcription factors. Compounds were chosen because of reported species differences in their interaction between the murine and human receptors (reviewed in ref. 6).

The application of mice humanized or nulled at the Car and Pxr gene loci also allowed a determination of the degree to which inducing agents act through CAR and/or PXR in regulating gene expression. In order not only to validate the functions of these receptors but also to investigate the crosstalk between them in regulating gene expression by different compounds, we used Cyp3a11 and Cyp2b10 as markers. These genes are known to be regulated in both CAR- and PXR-dependent fashion and demonstrate specificity toward the CYP substrates 7-benzyloxyquinoline (BQ) and pentoxyresorufin, respectively $(27,28)$.

When WT mice were treated with the putative human-specific PXR inducer RIF $(16,29)$ we unexpectedly found an induction of Cyp3a11 at higher doses (Figure 5A). This finding was reproducibly confirmed in repeated experiments. In the huPXR mice, a marked induction was also observed but at a much lower RIF dose, with effects observed at a dose of $3 \mathrm{mg} / \mathrm{kg}$ versus $20 \mathrm{mg} / \mathrm{kg}$ in the control animals. Therefore, in the equivalent range of standard oral dosing regimen in humans (300-600 mg per 70-kg humans), Cyp3a11 induction could only be observed in huPXR mice but not in the WT. No induction of Cyp3a11 expression measured by Western blot or enzyme activity was detected in the
PXR KO mice (Figure 5A). This observation demonstrates that the induction of Cyp3a11 by RIF is PXR mediated and also that PXR in the humanized mouse line is functional. The activation of PXR by lower doses of RIF in humanized compared with WT mice was also confirmed by BQ-activity measurements (Figure 5B). No differences in induction profiles of Cyp3a11 or BQ activity were observed between WT and humanized mice at high doses of RIF, e.g., $100 \mathrm{mg} / \mathrm{kg}$ (data not shown). In WT, huPXR, and PXR KO mice, no induction of Cyp2b10 expression was observed at any of the RIF doses tested, indicating that Cyp2b10 is not responsive to PXR activation by this inducer.

Treatment of WT and huPXR mice with the potent mouse inducer dexamethasone (DEX) $(14,30)$ resulted in a much more marked consistent induction in WT than in huPXR mice. At doses of up to $10 \mathrm{mg} / \mathrm{kg}$, induction of Cyp3a11 was only observed in WT but not huPXR mice (Figure 6A). However, at 30 and $60 \mathrm{mg} / \mathrm{kg}$, induction was also observed in humanized animals. It would therefore appear that there is a 10- to 30 -fold sensitivity difference between the MPXR and hPXR toward this compound. In PXR-null mice, the induction of Cyp3a11 was negligible and insignificant in repeated experiments and associated BQ activity was completely lost, demonstrating the pivotal role of this receptor in mediating this effect (Figure 6, B and C). Суp2b10 expression was also profoundly induced in WT and huPXR mice at all the DEX doses tested. The finding that this induction still occurred maximally in PXR-null mice (Figure 6, B and C) suggests that this involves a PXR-independent mechanism. Interestingly, DEX was also a potent inducer of Cyp2b10 in the CAR KO mouse (data not shown), suggesting that neither PXR nor CAR mediates the induction of this protein.

In addition to the experiments with RIF and DEX, we then carried out further experiments with clotrimazole and PCN as inducing agents. Clotrimazole was a potent inducer of Cyp3a11 and Cyp2b10 in both the WT and huPXR mice, and its potency as an inducer appeared essentially to be the same in both mouse strains. In the case of PCN, an induction of Cyp3a11 was already seen at a dose of $1 \mathrm{mg} / \mathrm{kg}$ in the WT mice, which was not observed in the 
A

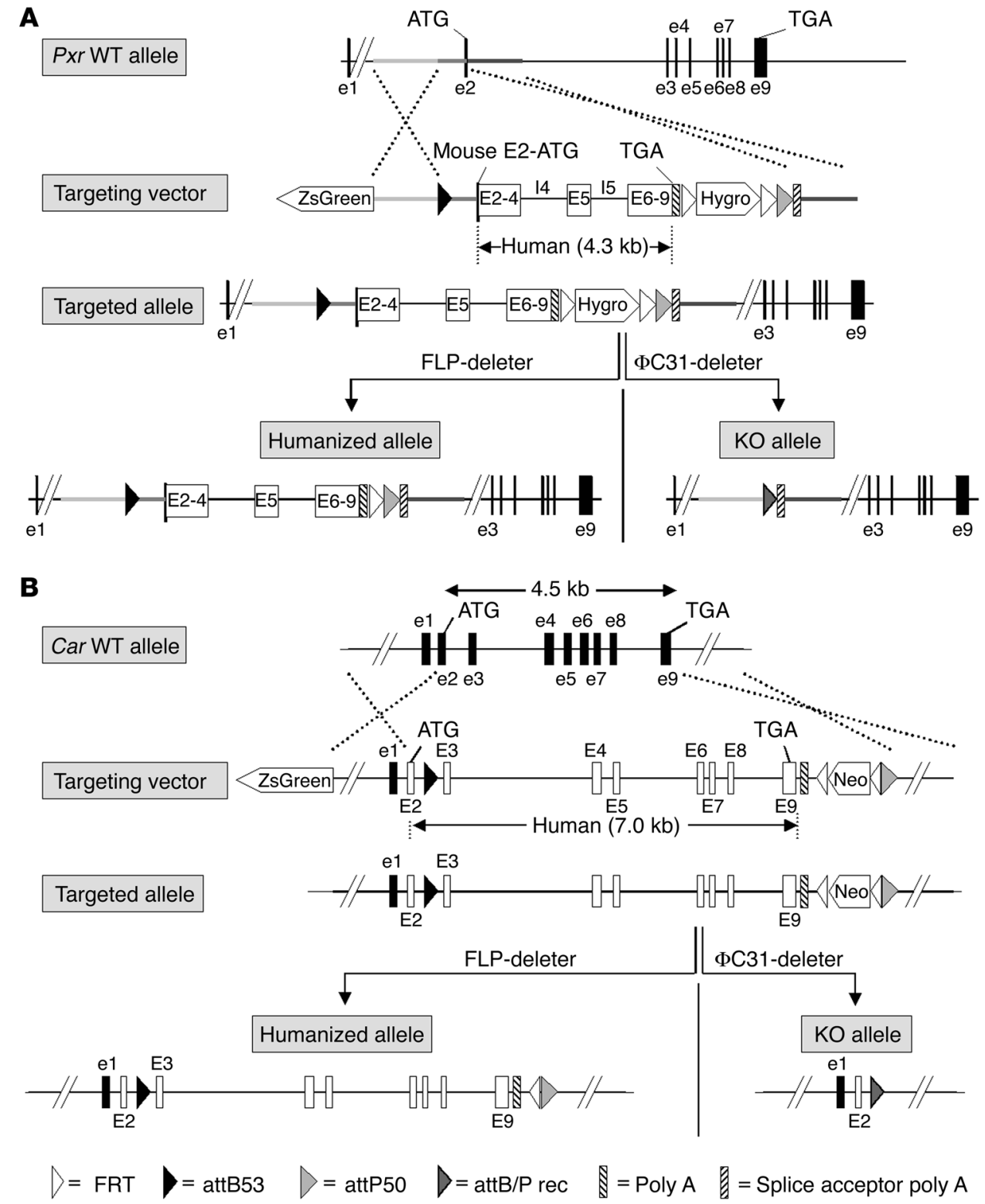

\section{Figure 2}

Strategy to generate huPXR, huCAR, and KO mice. (A) PXR: An $h P X R$ minigene containing a fusion of exons $2-4$, intron 4 , exon 5 , intron 5 , and a fusion of exons 6-9 was knocked in onto the translational start ATG of the mPxr WT gene in order to generate PXR-targeted mice. (B) CAR: The coding region of the $m$ Car WT gene was replaced with the genomic coding region of $h C A R$, including exons $2-9$, in order to generate CAR-targeted mice. In both cases, mouse exons are indicated in black and with lower-case letters; human exons are indicated in white and with upper-case letters. Targeted mice are crossed to a mouse strain expressing the FLPe recombinase to delete the hygromycin or neomycin selection cassette, respectively, and to generate huPXR or huCAR mice or to a $\phi$ C31 deleter strain to generate PXR or CAR KO mice. For the sake of clarity, sequences of the targeting vector are not drawn to scale.
huPXR mice, indicating a greater affinity of PCN for the mouse murine relative to the human receptor. At the doses used, PCN was not an inducer of Cyp2b10 (data not shown).

Functional analysis of the mouse models by using CAR activators. To further characterize the humanized mouse models, we carried out induction studies with compounds reported to exert their effects through their interactions, predominantly with CAR rather than PXR. To this end, we investigated the inducing properties of CITCO and TCPOBOP. CITCO has previously been reported to be a potent human-specific inducer of CYPs through the transcription factor CAR (13) and TCPOBOP as a murine-specific CYP inducer acting through this transcription factor $(14,15,31)$. Administration of CITCO to the mouse panel at a dose of $10 \mathrm{mg} / \mathrm{kg}$ for 3 days demonstrated a very minor induction of Cyp2b10 in the WT animals and a much more profound induction in huCAR animals (Figure 7, A and B). We observed some variability in the constitutive expression of Cyp2b10 in individual mice of the same genotype, and in the huCAR mice, there was a slight but consistent increase in constitutive Cyp2b10 levels compared with WT mice. This is similar to the increased expression of Cyp3a11 in PXR KO mice. The mechanism of this is unknown but the effect has been reported previously (32). The fact that the absolute level of Cyp2b10 expression following a CITCO treatment of $10 \mathrm{mg} / \mathrm{kg}$ was strongly elevated in huCAR mice but not in any of the other models tested, including the CAR-null and huPXR mice (Figure $7 \mathrm{~A}$ ), indicates that this induction is mediated only by hCAR, but not PXR. Associated with the induction of Cyp2b10 in the huCAR mice, a marked induction in pentoxyresorufin O-dealkylation was observed at all doses tested (Figure 7C). Using ranges of different doses, Cyp3a11 was also inducible in huCAR mice but not in the WT animals (Figure 7B). Furthermore, in the huPXR mice, no induction of hepatic Cyp3a11 expression was observed over background (data not shown), suggesting that the induction of this protein is also solely mediated by CAR. Considering the depen- 
A
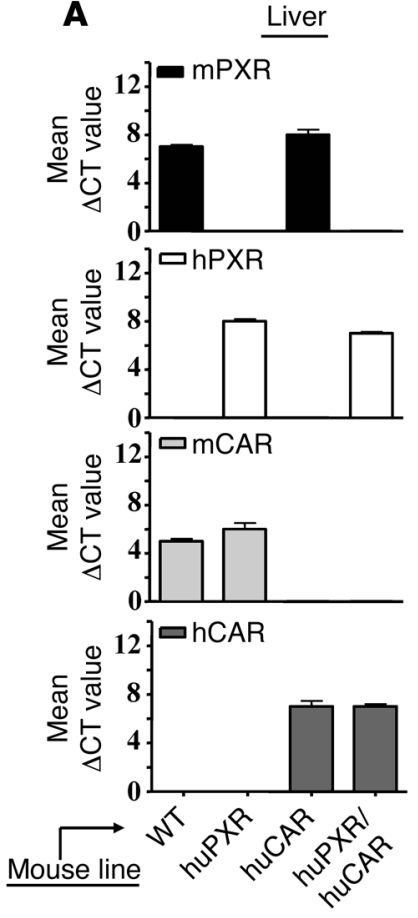

B
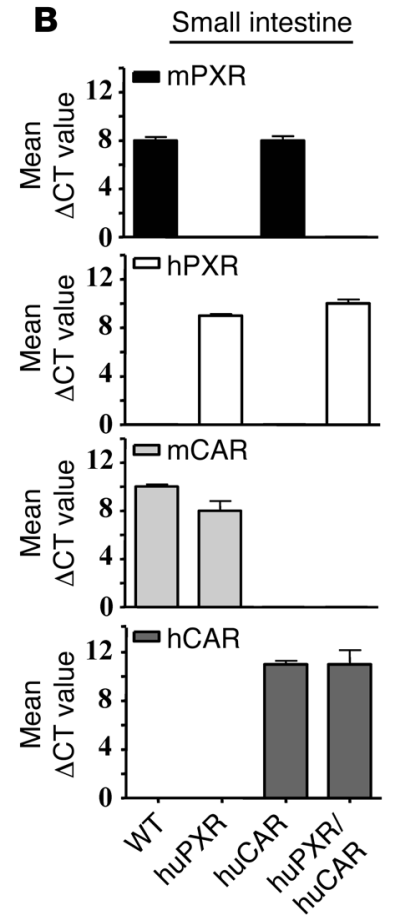

dence of Cyp2b10 and Cyp3a11 induction on CITCO concentration (Figure 7B), there appears to be an up to 40 -fold difference in the potency of CITCO in its capacity to activate hCAR as compared with mCAR. Interestingly, however, at the highest doses of CITCO, mCar can clearly be activated though not to the same extent as hCAR (Figure 7, B and C).

Administration of TCPOBOP to WT mice caused a profound induction of both Сyp2b10 and Сyp3a11, and a very slight induction of these proteins was also observed in the huCAR mice at a dose of $1 \mu \mathrm{g} / \mathrm{kg}$. These effects, particularly the induction in the WT animals, were completely lost in the CAR but not the PXR $\mathrm{KO}$ animals, demonstrating that these inductive effects are mediated by CAR and not by PXR (Figure 8A). The changes observed in Cyp2b10 protein were also reflected in the pentoxyresorufin activities (Figure 8C). Dose-response experiments demonstrated that even at doses of $0.3 \mathrm{mg} / \mathrm{kg}$, profound induction of Cyp $2 \mathrm{~b} 10$ was observed in WT but not in the huCAR animals (Figure 8B). In addition, induction of this protein was observed at high doses of TCPOBOP in the huCAR mice, but even at the highest doses, the induction of Cyp2b10 was much weaker than in the WT and was first observable at a dose of $1 \mathrm{mg} / \mathrm{kg}$. In summary, we confirmed that both huPXR and huCAR mice do express functional human receptors and that both lines show the expected humanized profile of interaction with selected ligands of PXR and CAR.

Generation and characterization of the PXR/CAR panel of mouse lines. Based on the genetic modifications described above, we generated a panel of mouse lines containing different alterations at the Pxr and Car locus. In addition to the huPXR, PXR KO, huCAR, and CAR KO mouse lines already described, this panel included all possible combinations of these modifications (huPXR/CAR KO, PXR $\mathrm{KO} /$ huCAR, PXR KO/CAR KO, and huPXR/huCAR). In order to demonstrate the validity of this approach, a basic characterization of the huPXR/huCAR mouse line was performed. First we asked whether the expression of both human receptors is maintained

\section{Figure 3}

$h P X R, m P X R, h C A R$, and $m C A R$ mRNA expression. RNA was isolated from untreated WT $(n=27)$, huPXR $(n=9)$, huCAR $(n=13)$, and huPXR/huCAR $(n=13)$ mice. $m P X R, h P X R, h C A R$, and $m C A R$ mRNA expression was analyzed by qPCR (TaqMan) in the liver (A) and small intestine (B). Values represent mean $\Delta \mathrm{Ct}$ values (Ct values for each gene of interest normalized to the Ct values for the internal standard $\beta$-actin) for all mice/genotypes. Error bars indicate SD in $\triangle C T$ values between animals of the same genotype.

in the huPXR/huCAR mouse line. To test this, TaqMan analysis was performed with CDNA obtained from liver and small intestine of huPXR/huCAR mice and results were compared with those obtained for the single-humanized mice. No difference in PXR and CAR expression levels could be detected in the single- and huPXR/ huCAR animals (Figure 3, A and B). Furthermore, as treatment of huPXR/huCAR mice with selected PXR and CAR agonists led to the same pattern of Cyp3a11 and Cyp2b10 activation as in the single-humanized mice (data not shown), we concluded that the expression of functional receptors is maintained.

Relevance of PXR and CAR for the induction of Cyp3a11 and Cyp2b10 by $P B$ in vivo. We then used the entire PXR/CAR panel to further assess whether the activation of hPXR and to a lesser extent MPXR by $\mathrm{PB}$ that has been observed in vitro (16) would have any significance for the regulation of Cyp3a11 or Cyp2b10 by this compound in vivo. In WT animals, administration of $\mathrm{PB}$ at $40 \mathrm{mg} / \mathrm{kg}$ markedly induced Cyp2b10 expression and to a lesser extent that of Cyp3a11 (Figure 9A). Induction of both these proteins was also observed in all huCAR mouse lines, demonstrating that the human receptor equally responds to $\mathrm{PB}$. However, in all mouse lines that carry a $\mathrm{KO}$ of CAR, the induction of Cyp2b10 and Cyp3a11 was strongly attenuated, whereas it was unaffected in PXR KO animals, indicating that CAR is the transcription factor that mediates induction of Cyp2b10 and Cyp3a11 (Figure 9A). The fact that induction of both Cyp3a11 and Cyp2b10 is also absent in the huPXR/CAR KO mice shows that hPXR is not able to compensate for the loss of CAR activity. These effects were also reflected in the measurement of BQ and pentoxyresorufin dealkylation (PROD) (Figure 9B).

Increased clearance of midazolam and bupropion in CITCO-treated huCAR mice. A mixture of midazolam and bupropion was administered i.p. to WT and huCAR mice on day 1 of the study. The same mice then received daily doses of the human-specific CAR activator CITCO on days 2-4 followed by another i.p. dose of the midazolam/bupropion mixture on day 5 . The pharmacokinetics of these Сур3a and Сyp2b substrates was determined before and after the treatment with the CAR activator in order to assess the CITCO-mediated changes in expression levels of Cyp $2 \mathrm{~b}$ and Cyp3a isoforms (Figure 10A). Both mouse lines demonstrated fast absorption of the substrates after the i.p. administration, which was not influenced by CITCO. No change in midazolam disposition was observed in the WT mice, while the AUC notably decreased in huCAR mice after CITCO treatment. Bupropion AUC was decreased on day 5 both in WT and transgenic animals, indicating induction of Сyp2b10. In order to demonstrate the differences in Cyp3a and Cyp2b elevation in WT and huCAR mice following the administration of CITCO, the ratios of the midazolam and bupropion AUCs before and after CITCO treatment were calculated using noncompartmental analysis (Figure 10B). This was based on the observation that after i.p. administration of an isoform-specific CYP substrate, the ratio of the expression level of 

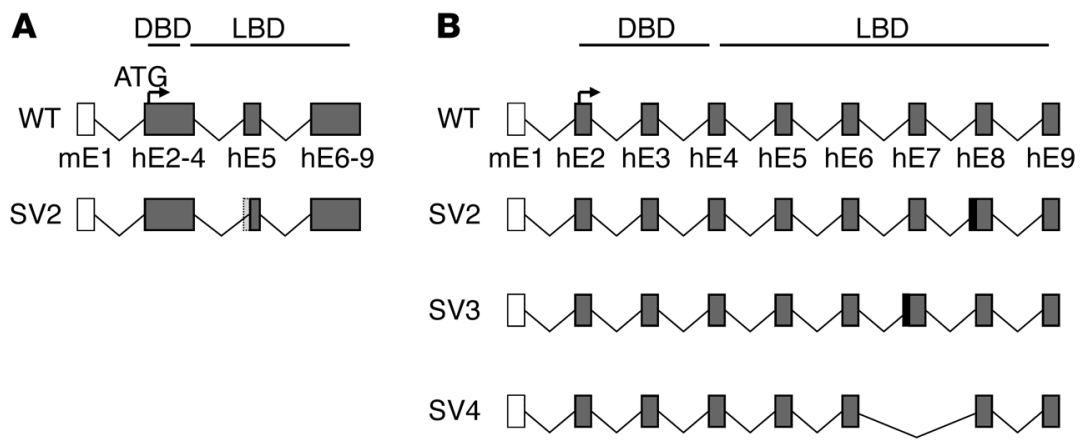

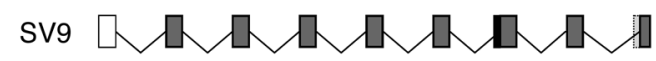

\section{Figure 4}

Isoforms corresponding to known human splice variants detected in the $(\mathbf{A})$ huPXR and $(\mathbf{B})$ huCAR mouse. Exons are presented as white (mouse) or dark gray (human) boxes. Deleted parts of exons are indicated as light gray, inserted parts as black boxes. The different isoforms are denominated according to their names in previous publications $(19,22)$. In all cases, the noncoding exon 1 is of mouse origin. In both huPXR and huCAR mice, further and yet unidentified splice variants presumably coding for nonfunctional receptors were detected at low abundance (not shown). DBD, DNA-binding domain; SV1-9, splice variants 1-9. the corresponding CYP before and after induction is inversely proportional to the ratio of the AUCs of the substrate $(33,34)$. For the WT mice, the midazolam AUC ratio was 1 , indicating no CITCOinduced changes in Сyp3a expression levels. In contrast, the Сyp3a level was elevated 3-fold in the huCAR mice after administration of CITCO, showing the higher sensitivity of the transgenic mice to this compound. In the case of bupropion, an increased AUC ratio could be observed in the WT mice, probably reflecting the minor induction of Cyp2b10 expression that we observed before (Figure 8A). However, the AUC ratio was notably higher in the huCAR mice, demonstrating the significantly stronger induction of Cyp2b10 expression by CITCO in the transgenic animals.

\section{Discussion}

The identification of PXR and CAR as nuclear receptors that regulate the expression of genes involved in the biotransformation and distribution of foreign chemicals in a ligand-dependent manner represented a major step forward in our understanding of the factors that control the activity of drug-metabolizing enzymes and transporters $(16,35-37)$. The marked species differences in the induction of phase I, II, and III enzymes in response to various compounds raise a difficulty in extrapolating metabolism data across species $(30,38-41)$. Taking into account the relevance of drug metabolizing enzyme and transporter induction or repression for drug safety and efficacy, these species differences clearly limit the significance of animal tests in respect to the situation in humans.

In order to evaluate the interaction of compounds with hCAR or hPXR in vitro, transactivation assays are routinely used (reviewed in ref. 6). The most common approach is to use reporter constructs that are transiently transfected into either continuously cultured cell lines or primary cells. As these tests can be applied in a high-throughput format, they are valuable tools to get a first indication of PXR or CAR interaction at an early stage. However, these approaches have limitations, as they depend on the cell line or primary culture

Figure 5 system used and the promoter/enhancer sequence of the reporter construct (6). More importantly, the absence of cell lines with significant hepatic functions means that the complex interactions of an in vivo system cannot be predicted.

Mice humanized for key regulatory proteins can overcome some of these limitations. For example, it was demonstrated that RIF only induced CYPs in mice expressing hPXR and not WT PXR (12). The opposite was the case when PCN was used. Furthermore, Ma et al. demonstrated a human-like profile of the drug-drug interaction in hPXR mice for RIF and midazolam (24).

In this paper, we describe the generation and characterization of a panel of new mouse models reflecting the PXR/CAR signaling pathway. We have generated single-humanized and $\mathrm{KO}$ mice of both receptors and all possible combinations thereof. This panel therefore constitutes a toolbox of mouse models to study in vivo the crosstalk of PXR and CAR and to evaluate their individual contribution to drug and chemical efficacy and safety in humans.

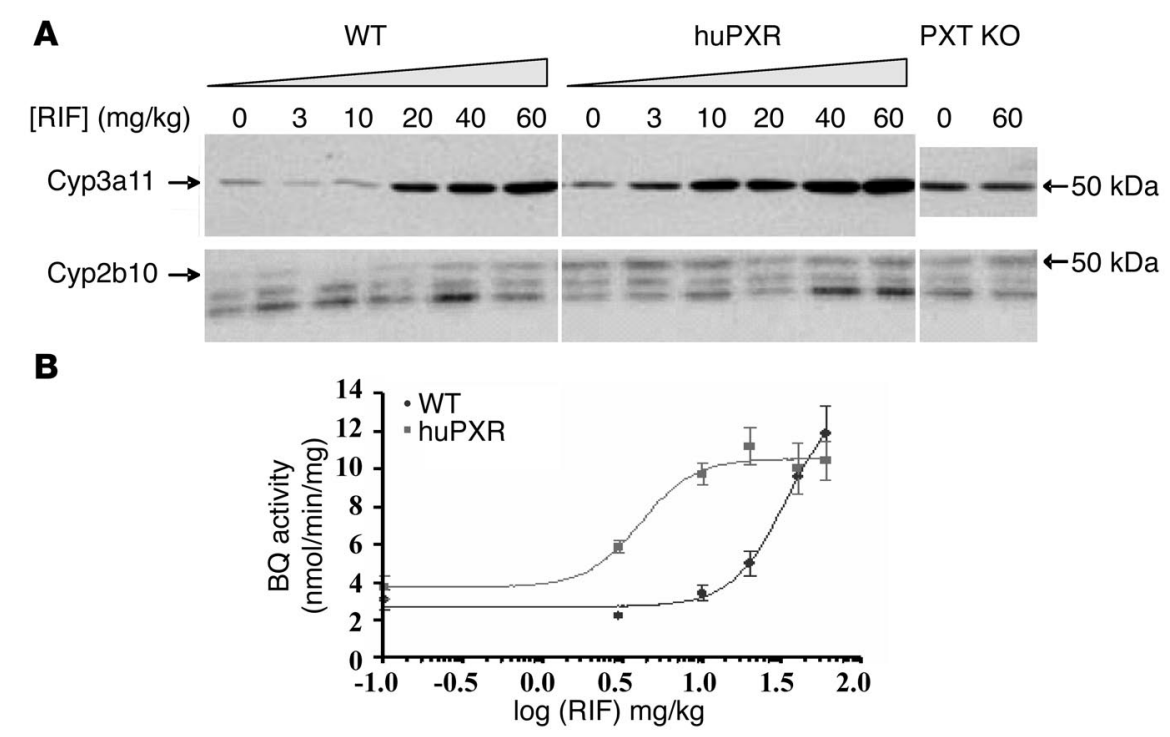

PXR-dependent induction of Cyp3a11 by RIF. (A) Pooled liver microsomes from huPXR, PXR $\mathrm{KO}$, and WT mice $(n=3)$ treated with either RIF or the vehicle were analyzed for Cyp3a11 and Cyp2b10 expression by immunoblotting. $5 \mu \mathrm{g}$ protein was loaded onto a $7.5 \%$ SDS-PAGE gel, and membranes were incubated with polyclonal Cyp3a11 and Cyp2b10 antibodies. (B) $\mathrm{BQ}$ assays were performed to investigate Cyp3a activity in pooled WT or huPXR mouse liver microsomes. Values are expressed as mean \pm SD. $n=3$ mice for all experiments. 
A

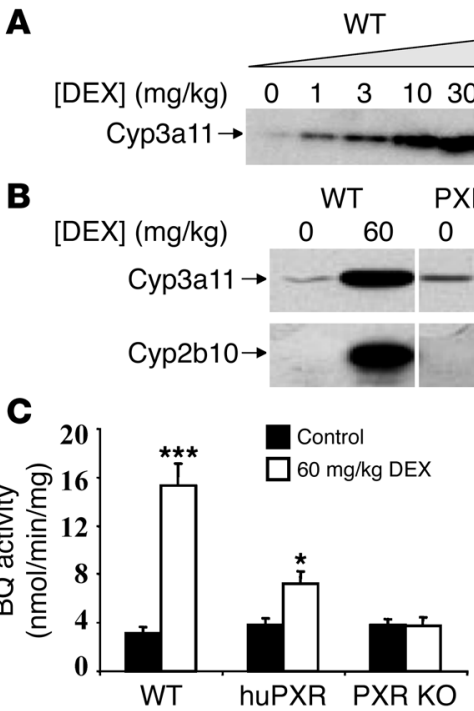

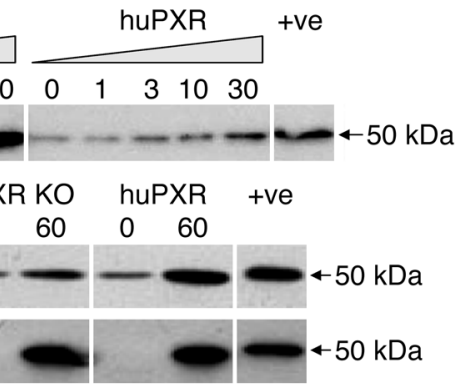

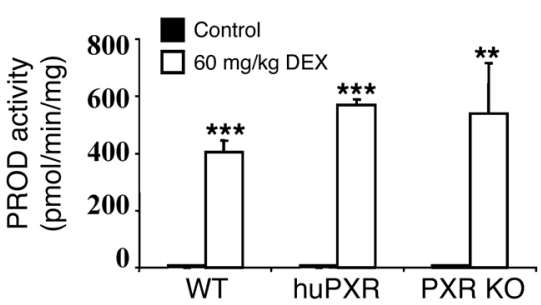

\section{Figure 6}

PXR-dependentinduction of Cyp3a11 by dexamethasone. (A and B) Pooled liver microsomes from huPXR, PXR $\mathrm{KO}$, and WT mice $(n=3)$ treated with either DEX or the vehicle were analyzed for Cyp3a11 and Cyp2b10 by immunoblotting. $5 \mu \mathrm{g}$ protein was loaded onto a $7.5 \%$ SDS-PAGE gel, and membranes were incubated with polyclonal Cyp3a11 and Cyp2b10 antibodies. (C) BQ and PROD assays were performed to investigate Сyp3a and Сур2b activities, respectively, in pooled WT, huPXR, or PXR KO mouse liver microsomes $(n=3$ mice). Values are expressed as mean $\pm \mathrm{SD} ; n=3$ mice for all experiments. Student's $t$ test (2-sided) was performed on the results. ${ }^{*} P<0.05$; ${ }^{* *} P<0.01 ;{ }^{* * *} P<0.001$, statistically different from control. +ve, His-tagged mouse Cyp2b10 or Cyp3a11 recombinant proteins.
The humanized receptor mice exhibited species-specific differences in the interaction with known drugs. In agreement with previous studies, huPXR was strongly activated by RIF but only weakly by DEX and PCN. In the case of huCAR mice, CITCO was a potent activator, while TCPOBOP barely activated the human receptor at all. Therefore, the humanized mice appear to reliably reflect the interaction of compounds with these human receptors.

Furthermore, we developed a pharmacokinetic approach that allows one to investigate species-specific differences in enzyme induction in huCAR and WT mice. Using this method, we could show that CITCO at a dose of $10 \mathrm{mg} / \mathrm{kg}$ induced Cyp3a expression in huCAR mice while no changes were observed in WT animals and also that Cyp2b induction was much more pronounced in the huCAR than in WT mice. Therefore, it was demonstrated that species specificities in CAR activation could be detected with this pharmacokinetic approach.

Our huPXR and huCAR mouse models are distinct from previously reported models in that we targeted the human transcription factors into the mPxr and $m$ Car gene loci. This means that the expression of the human receptors is controlled by the corresponding mouse promoters. Therefore, expression is not restricted to the liver but occurs in other organs where PXR and CAR are normally expressed. We have demonstrated this for the liver and small intestine, and PXR expression was detected in the kidney of huPXR mice by using quantitative PCR (data not shown). This is important in both pharmacokinetic and toxicological contexts. For example, in a recent study, it was shown in huPXR mice that rifaximin, a rifamycin

\section{Figure 7}

CAR-dependent induction of Cyp2b10 by CITCO. (A and B) Pooled liver microsomes from huCAR, CAR KO, PXR KO, huPXR, and WT mice $(n=3)$ treated with either CITCO or vehicle were analyzed for Cyp2b10 and Cyp3a11 expression by immunoblotting. $5 \mu \mathrm{g}$ protein was loaded onto a 7.5\% SDS-PAGE gel, and membranes were incubated with polyclonal Cyp2b10 or Cyp3a11 antibodies. (C) PROD assays were performed to investigate Cyp2b activity in pooled WT or huCAR mouse liver microsomes ( $n=3$ mice). Values are expressed as mean $\pm \mathrm{SD} ; n=3$ mice for all experiments. Student's $t$ test (2-sided) was performed. ${ }^{\star} P<0.05$; ${ }^{\star \star} P<0.01$; ${ }^{* \star *} P<0.001$, statistically different from control. analogue approved for the treatment of travelers' diarrhea, is a gutspecific hPXR ligand that significantly induces PXR target genes in the intestine but not in the liver of huPXR mice (42). Furthermore, it was shown that Pxr is expressed in rat brain capillaries, where it is involved in the regulation of Mdr1 (43). The potential importance of $\mathrm{Mdr} 1$ expression in the brain was illustrated by the finding that a 20- to 50-fold increase in the accumulation of cyclosporin A and digoxin in the brain is observed in Mdr1a-null mice (44). The lack of antibodies that allow the detection of the receptors in tissue samples currently prevents a direct confirmation of PXR and CAR protein expression throughout the body, and the assessment of their function in different organs requires further investigation.

The targeted integration of our expression constructs into the endogenous mouse loci ensures that all of the required promoter and enhancer elements for correct expression are present and that

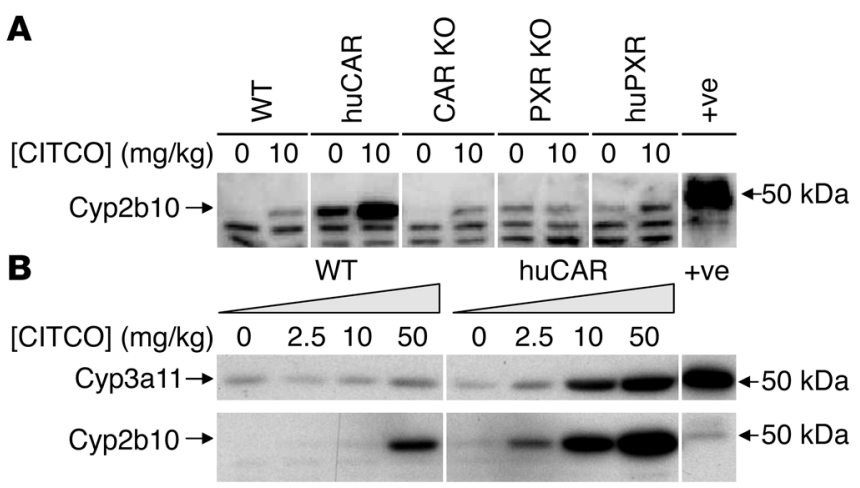

C

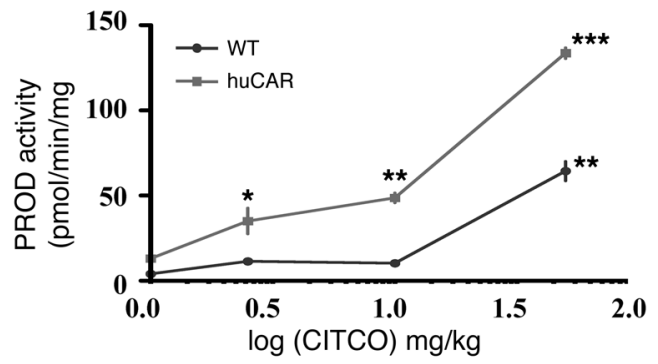




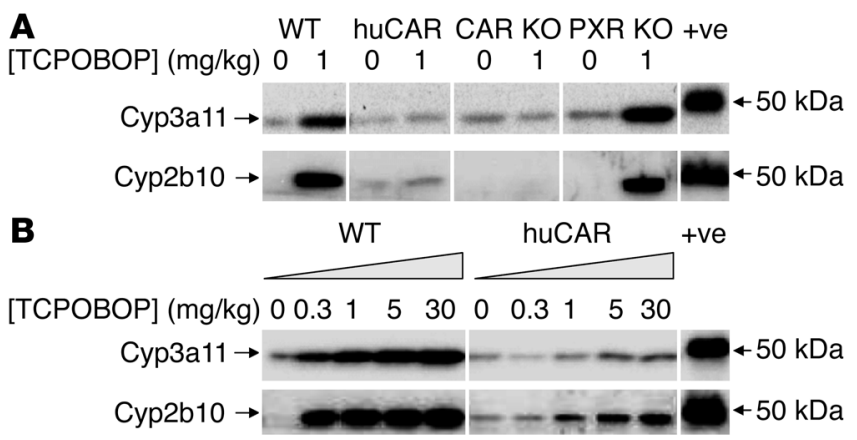

C

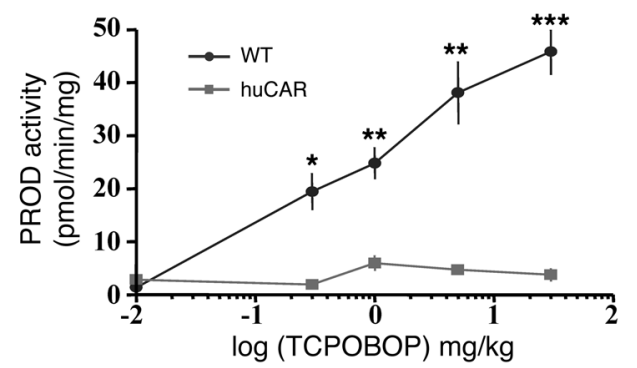

trans-acting factors of murine origin can accurately interact with the cis elements in the Car and Pxr promoters (e.g., HNF4). Additionally, the idiosyncratic positional effects that are frequently observed with randomly integrated transgenes are excluded by this approach. In support of this strategy, we found that mRNA expression levels of $P X R$ and CAR in the humanized mice are comparable to those of the mouse mRNAs in WT animals (Figure 3) and are in good concordance with levels expressed in human liver samples (data not shown). Due to the fact that ligand-dependent receptor activation doesn't work through an all-or-nothing mechanism but is rather a gradual process depending on the dosing regimen, physiological expression

\section{Figure 8}

CAR-dependent induction of Cyp2b10 by TCPOBOP. (A and B) Pooled liver microsomes from huCAR, CAR KO, PXR KO, and WT mice $(n=3)$ treated with either ТСРОВОР or vehicle were analyzed for Сyp2b10 and Cyp3a11 expression by immunoblotting. $5 \mu \mathrm{g}$ protein was loaded onto a $7.5 \%$ SDS-PAGE gel, and membranes were incubated with polyclonal Cyp2b10 and Cyp3a11 antibodies. (C) PROD assays were performed to investigate Cyp2b activity in pooled WT or huCAR mouse liver microsomes $(n=3)$. Values are expressed as mean $\pm \mathrm{SD} ; n=3$ mice for all experiments. Student's $t$ test (2-sided) was performed. ${ }^{\star} P<0.05 ;{ }^{* \star} P<0.01 ;{ }^{* \star *} P<0.001$, statistically different from control.

levels are critical for an accurate in vivo analysis of receptor interaction with a compound. For example, in contradiction with the consideration of RIF as a human-specific PXR activator, we found a reproducible induction of Cyp3a11 at high doses of this inducer (Figure 5, A and B). This observation was missed by studies in which only low doses were used. As similar findings were made for all other compounds tested, strict species-specific interaction of a compound with PXR or CAR seems to be an exception rather than a rule.

Another finding from our study was that the maximum induction of Cyp3a11 was equivalent at high RIF doses in huPXR and WT mice, while the magnitude of Cyp2b10 induction by TCPOBOP in the huCAR mice never reached that of the WT animals. This observation could be explained by distinct mechanisms of receptor activation by these compounds, such that only the different binding affinities of MPXR and hPXR for RIF account for the differential induction of Cyp3a11 in huPXR and WT mice, whereas TCPOBOP may induce an alternative conformational change in the human relative to the mCAR receptor. However, further experiments are required to determine whether this assumption is correct.

As an alternative to expressing full-length CAR and PXR cDNAs, we included genomic sequences in our targeting constructs. In the case of PXR, we retained introns 4 and 5 of the human gene, as this
Figure 9

CAR-dependent induction of Cyp2b10 and Cyp3a11 by PB. (A) Pooled liver microsomes from huPXR, PXR KO, huCAR, CAR KO, hUPXR/hUCAR, PXR KO/ CAR KO, huPXR/CAR KO, PXR $\mathrm{KO} /$ huCAR, and WT mice $(n=3)$ treated with either PB or vehicle were analyzed for Cyp2b10 and Cyp3a11 expression by immunoblotting. $5 \mu \mathrm{g}$ protein was loaded onto a $7.5 \%$ SDS-PAGE gel, and membranes were incubated with polyclonal Cyp2b10 and Cyp3a11 antibodies. (B) PROD and BQ assays were performed to investigate Cyp2b and Сyp3a activities, respectively, in pooled mouse liver microsomes from each mouse line indicated above ( $n=3$ mice). Values are expressed as mean \pm $\mathrm{SD} ; n=3$ mice for all experiments. Student's $t$ test (2-sided) was performed. ${ }^{*} P<0.05$; ${ }^{*} P<0.01$; ${ }^{* * *} P<0.001$, statistically different from control.
A

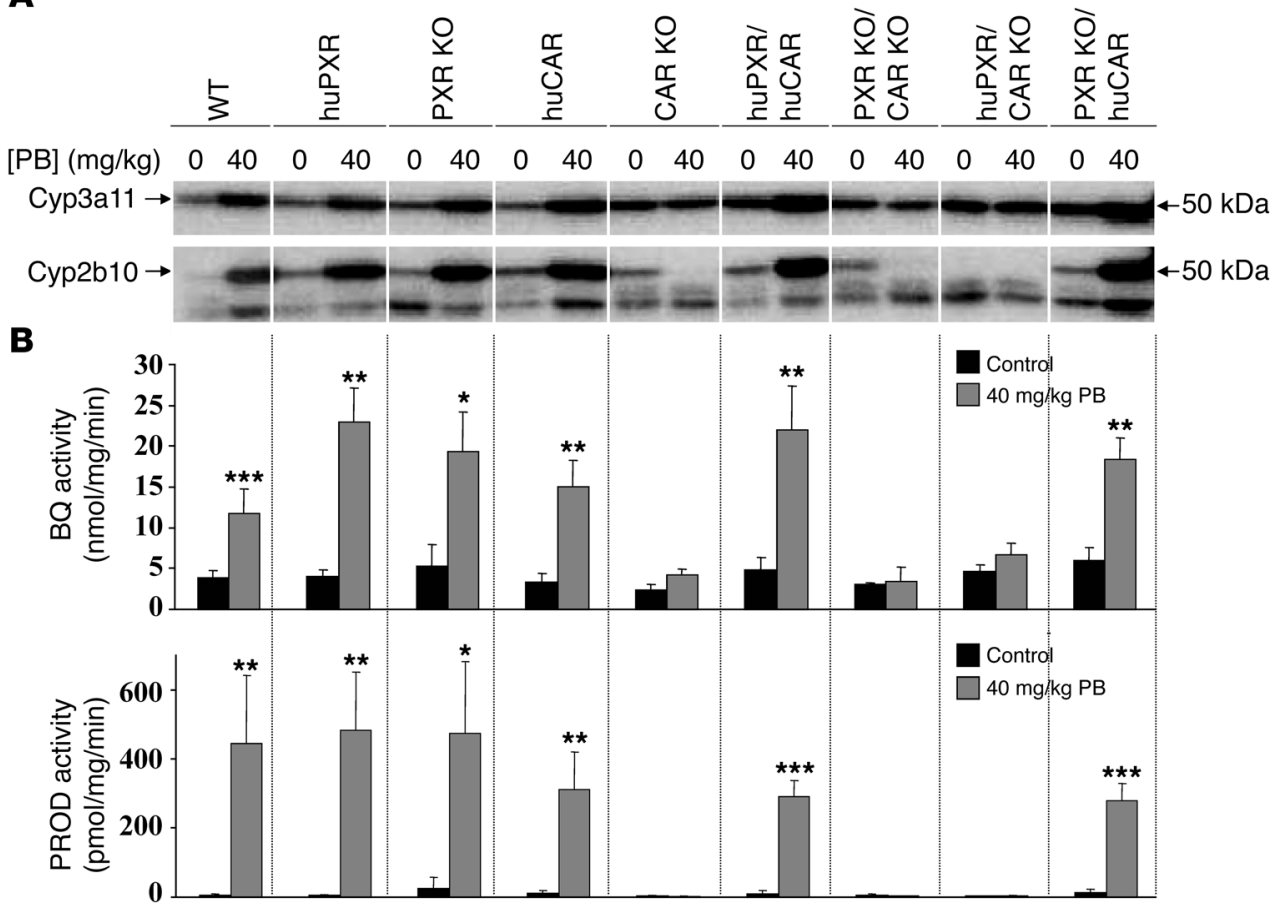


A
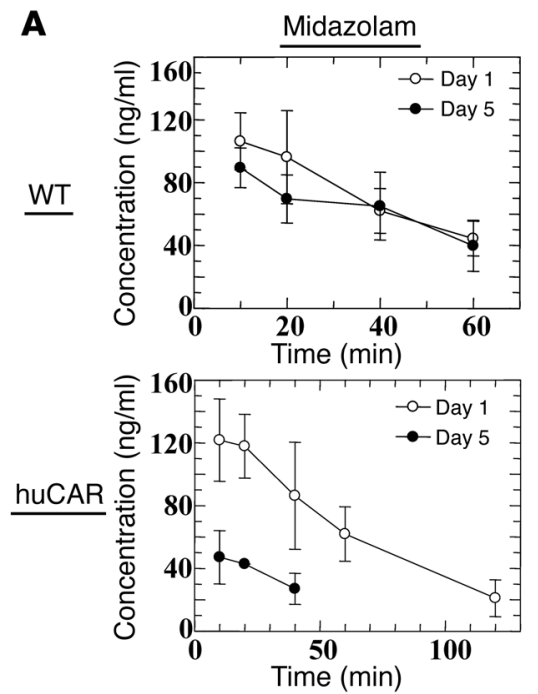

B

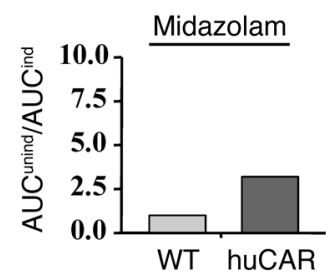

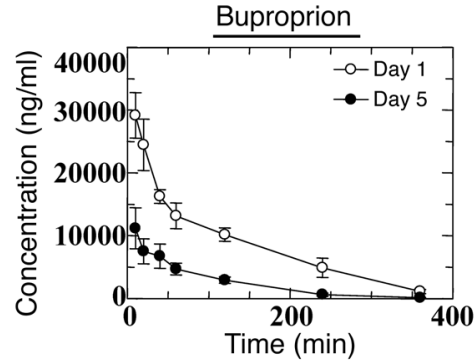

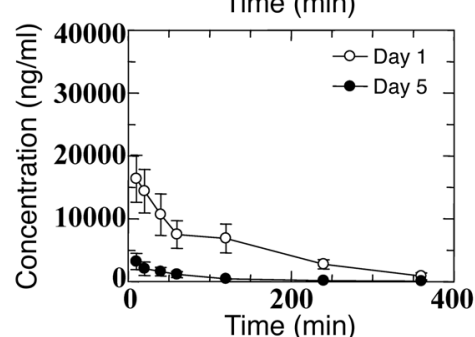

Buproprion

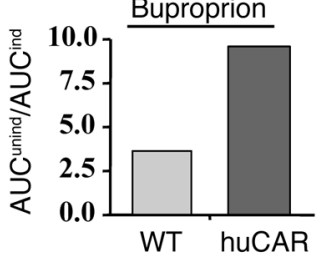

\section{Figure 10}

Midazolam and bupropion pharmacokinetics in CITCO-treated WT and huCAR mice. (A) Midazolam and bupropion pharmacokinetics in WT and huCAR mice before (day 1 ) and after (day 5) treatment with $10 \mathrm{mg} / \mathrm{kg}$ of 3 daily i.p. doses of CITCO. Values represent the concentration of parent drug in plasma $(\mathrm{ng} / \mathrm{ml})$ as mean of 4 mice. Error bars describe SD in either midazolam or bupropion plasma concentrations. (B) Ratios of the average midazolam and bupropion AUCs calculated from the corresponding pharmacokinetics of 4 mice per compound before (unind) and after (ind) administration of CITCO. genomic region is subject to important alternative splicing events that alter the LBD (19). At least the 2 most abundant splice isoforms found in humans, coding for the WT protein of 434 amino acids and a truncated protein lacking 37 amino acids of the LBD (called hPXR. 2 or hPXR SV2, respectively), were expressed in the huPXR mouse (Figure 4A). As the LBD is markedly changed in SV2 and was reported to show a different activation profile with respect to RIF, it is possible that this splice variant contributes to the functional diversity of $\operatorname{hPXR}(25,26)$. In the case of CAR, all human introns between the coding exons 2 and 9 were incorporated in the targeting vector. As a consequence, all 4 human splice variants described previously (21) were identified in the huCAR mouse. Importantly, these variants encode functional proteins with altered LBDs. For example, SV2 has a 5-amino acid insertion at exon 8, which invokes a ligand-dependent instead of a constitutive interaction with coact- ivators, and the SV3 isoform, with a 4-amino acid insertion at exon 7 , differentially transactivates target gene promoters, respectively $(22,45)$. Therefore, expression of these splice isoforms may be of significant importance in hCAR function.

The low genetic complexity of our models allowed us to develop a combinatorial approach to generate mice humanized for multiple pathways of drug metabolism. We believe this is important due to the complex network of these pathways and in the light of the crosstalk between them. To this end, we generated a panel of PXR/ CAR-modified mice, including huPXR, PXR KO, huCAR, and CAR $\mathrm{KO}$ single-modified mouse lines and all possible combinations of these. To our knowledge, this is the first report of huPXR/huCAR mice. One major strength of this panel is discriminating between PXR- and CAR-mediated effects by uncovering the crosstalk between these 2 receptors and evaluating the significance of these

\section{Table 1}

Determination of genotypes

\begin{tabular}{|c|c|c|c|}
\hline PCR type & Primers & Expected fragment(s) & Detected allele \\
\hline PXR WT & $\begin{array}{l}\text { 5'-GCTTCTCATTTCTCCCTCCTG; } \\
\text { 5'-TGATCCTTTCCTGGGCAGC }\end{array}$ & $700 \mathrm{bp}$ & Detects WT mPXr \\
\hline hPXR & $\begin{array}{l}\text { 5'-GCTTCTCATTTCTCCCTCCTG; } \\
\text { 5'-CTCCGACTTCCTCATCTGCG }\end{array}$ & $386 \mathrm{bp}$ & $\begin{array}{l}\text { Detects humanized } P X R \text { in both } \\
\text { FLP-deleted and -undeleted animals }\end{array}$ \\
\hline PXR KO & $\begin{array}{l}\text { 5'-CCTCCTCACTACTCTCCCAGC; } \\
\text { 5'-GTACCAGCAAGTGCGTGAAGG }\end{array}$ & $\begin{array}{l}1274 \mathrm{bp}(\mathrm{WT}) \\
944 \mathrm{bp}(\mathrm{KO})\end{array}$ & $\begin{array}{l}\text { Detects } \phi C 31 \text {-mediated deletion of } P X R \\
\text { in KO mice (944 bp) and WT PXr (1274 bp) }\end{array}$ \\
\hline CAR WT & $\begin{array}{l}\text { 5'-CTCAACTCCTCCCACATTCAG; } \\
\text { 5'-GCAAACGGACAGATGGGAC }\end{array}$ & $663 \mathrm{bp}$ & Detects WT mCar \\
\hline hCAR & $\begin{array}{l}\text { 5'-CTCAACTCCTCCCACATTCAG; } \\
\text { 5'-TGCTCTTGACTAATGGGCTG }\end{array}$ & 369 bp & $\begin{array}{c}\text { Detects humanized } C A R \text { in both FLP-deleted } \\
\text { and -undeleted animals }\end{array}$ \\
\hline CAR KO & $\begin{array}{l}\text { 5'-CTCAACTCCTCCCACATTCAG; } \\
\text { 5'-TCCCATCCCCTGTGTTTCC }\end{array}$ & 559 bp & Detects $\phi \mathrm{C} 31$-mediated deletion of $C A R$ in $\mathrm{KO}$ mice \\
\hline
\end{tabular}


interactions in vivo. For example, we have shown that the induction of Cyp3a11 by DEX is mediated primarily by PXR, with the murine receptor being most sensitive, whereas Cyp2b10 induction is independent of both PXR and CAR and could be mediated by the glucocorticoid receptor. Furthermore, although $\mathrm{PB}$ is reported to activate PXR in vitro (16), our studies on the PXR/CAR panel suggest that the PB-mediated activation of Cyp3a11 and Cyp2b10 in vivo is CAR-dependent, with PXR playing at best a minor role. This demonstrates that the in vitro interaction of a compound with PXR and CAR does not necessarily reflect its in vivo significance. The above examples demonstrate why the use of a combination of CAR and PXR mice adds markedly to the elucidation of how interactions with these transcription factors can affect drug disposition and toxicity in humans.

In summary, there are many applications for mice nulled and/or humanized for single or multiple transcription factors involved in drug disposition in humans. One of our ongoing goals in this project is to further increase the value of these models by introducing human phase I, II, and III genes onto the hCAR/hPXR background.

\section{Methods}

Animal husbandry. Mice were kept in Tecniplast Sealsafe microisolator cages. Food and water were available ad libitum. Light cycles were on a 13:11-hour light/dark cycle with the light phasing starting at 0600 hours. Temperature and relative humidity were maintained between $21^{\circ} \mathrm{C}$ and $23^{\circ} \mathrm{C}$ and $45 \%$ and $65 \%$.

Construction of PXR- and CAR-targeting vectors: PXR. A 6-kb genomic sequence within intron 1 of the $m P x r$ gene comprising 6.5 - to $0.5-\mathrm{kb}$ upstream of the translational start site and 4-kb 3 ' to exon 2 was subcloned by red/ET recombineering (46) and used as targeting arms for homologous recombination. The remaining $0.5 \mathrm{~kb}$ of intron 1 and exon $2 \mathrm{up}$ to the translational start ATG of $m P x r$ and exons 2-4 from a cDNA clone of the LPXR gene were amplified by PCR. Both fragments were used in a fusion PCR, so that the translational start ATG of the mPxr gene was fused to the coding sequences of exons 2-4 of $h P X R$, whereby the start CTG of the $h P X R$ gene (36) was deleted. By subsequent steps of regular PCR and fusion PCR cloning using a human BAC and PXR cDNA, a chimeric construct comprising mPxr start ATG and hPXR exons 2-4, intron 4, exon 5, intron 5, and exons 6-9, including the translational stop site, was generated. Finally, the 5 ' homology arm, chimeric humanization fragment, and the 3 ' homology arm were subcloned into a predesigned vector, carrying $53 \mathrm{bp}$ and 50 bp of the $\phi C 31$ recognition sites attB and attP, respectively (47), an SV40 poly(A) signal, a self-constructed splice acceptor site followed by a poly(A) signal, an flipase recombinase (FLP) recognition target-flanked hygromycin expression cassette, and a ZsGreen expression cassette, to give rise to the targeting vector depicted in Figure 2A.

Construction of PXR- and CAR-targeting vectors: CAR. $4.64 \mathrm{~kb}$ of genomic sequence of the $m$ Car gene immediately $5^{\prime}$ of the translational start ATG and $4.61 \mathrm{~kb} \mathrm{3} 3^{\prime}$ to exon 9 were subcloned by subsequent steps of PCR cloning from a mouse BAC and used as targeting arms for homologous recombination. The 5' homology arm was fused to a PCR amplified fragment of CAR exon 2 and 253 bp of the $5^{\prime}$ part of intron 2 from a human BAC, such that the translational start ATG of the $m$ Car gene was fused to the coding sequences of exon 2 of $h C A R$, whereby the start ATG of the $h C A R$ gene was deleted. 228 bp of the $3^{\prime}$ part of human intron 2 up to exon 9 including the translational stop site and 182-bp $3^{\prime}$ UTR was assembled as a separate fragment by consecutive steps of PCR cloning. Finally, the $5^{\prime}$ homology arm, including the coding region of hCAR exon 2 and the $5^{\prime}$ part of intron 2 , the fragment containing the $3^{\prime}$ part of human intron 2 and exons $3-9$, and the $3^{\prime}$ homology arm, was subcloned into a predesigned vector, carrying the $\phi \mathrm{C} 31$ recognition sites attB53 and attP50, an SV40 poly(A) signal, an FLP recognition target-flanked neomycin expression cassette, and a ZsGreen expression cassette, to give rise to the targeting vector depicted in Figure 2B.

Generation and molecular characterization of targeted ES cells: PXR. Culture and targeted mutagenesis of ES cells were carried out as previously described (48). The targeting vector was linearized with NotI and electroporated into a C57BL/ 6 mouse ES cell line. From 125 hygromycin-resistant and fluorescence-negative ES cell colonies screened by standard Southern blot analyses, 97 correctly targeted clones were identified. Six of these were expanded and further analyzed by Southern blot analyses with different suitable restriction enzymes, $5^{\prime}$ and $3^{\prime}$ external probes, and an internal hygromycin probe. Out of 6 clones, 5 were confirmed as correctly targeted at both homology arms and didn't carry additional random integrations (data not shown).

Generation and molecular characterization of targeted ES cells: CAR. The targeting vector was linearized with NotI and electroporated into one of the correctly targeted PXR ES clones described above. From 165 G418-resistant and fluorescence-negative ES cell colonies screened by standard Southern blot analyses, 41 correctly targeted clones were identified. Three out of 3 clones that were expanded and further analyzed by Southern blot analyses as described above were confirmed as correctly targeted at both homology arms and didn't carry additional random integrations (data not shown).

Generation and molecular characterization of huPXR and huCAR mice and KOs. One of the PXR single- and PXR/CAR double-targeted ES cell clones described above was expanded, injected into $B A L B c$-blastocysts, and transferred into foster mothers as previously described (48). Litters from these fosters were visually inspected, and chimerism was determined by hair color. Highly chimeric animals were used for further breeding in a C57BL/ 6 genetic background. Selection markers were removed in vivo by crossing to an efficient FLP-deleter (FLPe-deleter) strain carrying a transgene that expresses FLPe in the germ line. KOs were generated by crossing chimeras to a mouse strain expressing the $\phi \mathrm{C} 31$ recombinase in the germ line (Figure 2, A and B). Both FLPe and $\phi \mathrm{C} 31$ deleter have been generated in house on a C57BL/6 genetic background. The PXR single-targeted ES cell clone was used to generate huPXR and KO animals, and the PXR/CAR doubletargeted ES cell clone was used to generate huCAR and KO animals as well as huPXR/huCAR mice. The genotype of humanized and KO animals was determined by combination of the PCRs listed in Table 1 . The above procedures and additional breeding steps resulted in the following mouse lines: huPXR, huCAR, PXR KO, CAR KO, huPXR/huCAR, double-KO PXR and CAR (PXR KO/CAR KO), huPXR/CAR KO, and PXR KO/huCAR.

Mouse lines used for validation experiments. Homozygously humanized and KO mice for PXR and CAR were used for all studies. In the case of humanized mice, the hygromycin and neomycin selection cassettes, respectively, were removed by FLP-mediated deletion. WT C57BL/ 6 animals of the same genetic background and age purchased from Harlan were used for control experiments.

Animals and treatments. All animal procedures were carried out under a United Kingdom Home Office license, and all animal studies were approved by the Ethical Review Committee, University of Dundee. Male, sexually mature huPXR, huCAR, CAR KO, PXR KO, huPXR/huCAR, and WT (WT, C57BL/6J) mice were dosed daily by i.p. injection with either the vehicle, $\operatorname{RIF}(1,3,10,20,40,30$, or $60 \mathrm{mg} / \mathrm{kg} / \mathrm{d}), \operatorname{DEX}(1,3,10,30$, or $60 \mathrm{mg} / \mathrm{kg} / \mathrm{d})$, CITCO $(2.5,10$, or $50 \mathrm{mg} / \mathrm{kg} / \mathrm{d}$ ), PCN (1 or $3 \mathrm{mg} / \mathrm{kg} / \mathrm{d}$ ), clotrimazole ( 3 or $30 \mathrm{mg} / \mathrm{kg})$, or $\mathrm{PB}(40 \mathrm{mg} / \mathrm{kg} / \mathrm{d})$ for 4 days and sacrificed 24 hours after the last dose. ТСРОВОР $(0.3,1,5$, or $30 \mathrm{mg} / \mathrm{kg} / \mathrm{d})$ was administered by single i.p. injection, and the animals were terminated after 24 hours.

Quantitative RT-PCR. $h P X R, m P X R, b C A R$, and $m C A R$ RNA were analyzed by quantitative RT-PCR (qRT-PCR) (TaqMan). Total RNA was prepared from small intestine using TRIzol reagent (Invitrogen) and from the liver using the QIAGEN RNA Mini Kit. cDNA was synthesized from $1 \mu \mathrm{g}$ 
total RNA using the Quantiscript Reverse Transcriptase Kit (QIAGEN). Primers used were from the following assay-on-demand kits: hPXR Hs00243666_ml, mPXR Mm00803092_ml, mCAR Mm004437986_m1, hCAR Hs00231959_m1, and $\beta$-actin Mm00607939_m1 (Applied Biosystems). qRT-PCR reactions were performed using TaqMan Universal PCR Mastermix in an ABI PRISM 7000 Sequence Detection System (Applied Biosystems). Data were analyzed using comparative cycling time methodology, in which fluorescent output, measured as $\mathrm{Ct}$, was directly proportional to input cDNA concentration. A Ct value of 40 was interpreted as absence of gene expression, whereas Ct values in the range of 35 to 40 were interpreted as being at the limit of detection of the TaqMan and therefore not quantitatively analyzed. Input cDNA concentrations were normalized to murine $\beta$-actin. Values represent mean $\Delta \mathrm{Ct}$ values (Ct values for each gene of interest normalized to the $\mathrm{Ct}$ values for the internal standard $\beta$-actin) for all mice/genotype.

Sequencing analysis. RT-PCR was performed using RNA isolated from a huPXR mouse and huCAR mouse using oligos to amplify the potential fulllength transcript. The oligos used were as follows: P6-F, 5'-GGAATTCAACATGGAGGTGAGACCCAAAG-3'; hPXR-REV_3, 5'-TCAGCTACCTGTGATGCCGAACAAC-3'; CAR-F, 5'-CTCAAGGAAAGCAGGGTCAGC-3'; P-12F, 5'-GGATCCACCATGGCCAGTAGGGAAGATGAG-3'; and P-12R, 5'-TCAGCTACCTGTGATGCCGAACAAC-3'. One-step RT-PCR reaction was conducted using Superscript RT-PCR Kit (Invitrogen). Sequence analysis was performed by Lark Technologies Ltd. Alignments were performed using TCoffee (http://tcoffee.vital-it.ch/cgi-bin/Tcoffee/tcoffee_cgi/index. cgi) and Contig express and Align-X programs of Vector NTI8 software.

Hepatic microsomal preparation. Mouse livers used were freshly harvested, blotted, and weighed. The liver was scissor-minced in ice-cold $\mathrm{KCl}(1.15 \%$, $\mathrm{w} / \mathrm{v})$, then homogenized in ice-cold SET buffer $(0.25 \mathrm{M}$ sucrose, $5 \mathrm{mM}$ EDTA, and $20 \mathrm{mM}$ Tris-HCL, pH 7.4) to make a $10 \%(\mathrm{w} / \mathrm{v})$ homogenate solution ( $9 \mathrm{ml} \mathrm{SET}$ buffer $/ 1 \mathrm{~g}$ liver). Microsomes were prepared by centrifugation first at $6441 \mathrm{~g}$ (Heraeus 6445 rotor/Sorvall legend RT centrifuge) for 10 minutes at $4^{\circ} \mathrm{C}$; then the supernatant was spun at $13864 \mathrm{~g}$ (Sorvall F28/13 rotor) for 15 minutes at $4^{\circ} \mathrm{C}$. The resulting supernatant was spun at $82187 \mathrm{~g}$ (Sorvall F28/13 rotor) for 90 minutes at $4^{\circ} \mathrm{C}$, and the microsomal pellets were resuspended in ice-cold SET buffer and stored at $-70^{\circ} \mathrm{C}$.

Immunoblot analysis. For Western blot analysis, microsomal protein $(5 \mu \mathrm{g})$ from individual or pooled $(n=3)$ mouse samples was separated by SDS-PAGE, electrophoretically transferred to nitrocellulose membranes, and probed using an anti-rat CYP3A1 polyclonal $\mathrm{Ab}$ and antirat CYP2B1/2 polyclonal Ab (Biomedical Research Centre, University of Dundee) (49) at 1:2000 dilutions. The detection of mouse Cyp3a11 or Cyp2b10 by the corresponding rat antibodies was determined by Western blots using recombinant Cyp3a11 and Cyp2b10 proteins (Figure 6, A and B; Figure 7, A and B; and Figure 8, A and B). The secondary antibody was anti-rabbit HRP conjugate, used at a dilution of 1:2000 (GE Healthcare).
Detection of immunoreactive proteins was performed by an enhanced chemiluminescence blot detection system (Amersham Biosciences).

CYP enzyme activity assays. Activity of Cyp2b10 in hepatic microsomes was monitored using the preferred Cyp2b10 substrate, pentoxyresorufin. The dealkylation of this compound was monitored in a $1 \mathrm{~cm}^{3}$ cuvette containing $66 \mathrm{mM}$ Tris- $\mathrm{HCl}$ buffer, $\mathrm{pH} 7.4$, microsomes $(20-50 \mu \mathrm{g})$, and $1 \mathrm{mM}$ pentoxyresorufin. The reactions were initiated by the addition of $50 \mu \mathrm{M}$ $\mathrm{NADPH}$, and the rate of fluorescence was measured. As an internal control, each microsomal mixture was spiked with $10 \mu \mathrm{M}$ resorufin.

Dealkylation of BQ $(20 \mathrm{mM})$, a Cyp3a11 preferred substrate, was measured in liver microsomes $(20-50 \mu \mathrm{g})$ suspended in $66 \mathrm{mM}$ Tris- $\mathrm{HCl}$ buffer, $\mathrm{pH}$ 7.4. Assays were initiated by the addition of $50 \mu \mathrm{M}$ NADPH to the microsomal reaction and followed using a Hitachi F-4500 fluorescence spectrophotometer with FL Solution 2.0 software. 7-Hydroxyquinoline (7-HQ, $1 \mathrm{mM}$ ) was used as the internal standard.

Protein concentrations were measured using a modified version of the method of Lowry (50) with bovine serum albumin standards. Values were calculated and expressed as $\mathrm{nmol} / \mathrm{min} / \mathrm{mg}$ protein or $\mathrm{pmol} / \mathrm{min} / \mathrm{mg}$ protein for the BQ and PROD assays, respectively.

Midazolam and bupropion pharmacokinetic following treatment with CITCO. A mixture of bupropion $(87 \mathrm{mg} / \mathrm{kg})$ and midazolam $(0.9 \mathrm{mg} / \mathrm{kg})$ was administered i.p. to the WT and huCAR mice (groups of 4 mice), and $10 \mu \mathrm{l}$ blood samples were collected at 10, 20, 40, 60, 120, 240, and 360 minutes. The mice then received 3 daily i.p. doses of CITCO $(10 \mathrm{mg} / \mathrm{kg})$. On day 5 of the study, the midazolam and bupropion mixture was administered again and blood samples collected for pharmacokinetic analysis. Concentrations of midazolam and bupropion in the whole-blood samples were determined using reverse-phase HPLC (Waters Corp.) with tandem mass spectrometric detection (LC-MS/MS).

Statistics. Statistical significance was assessed to determine differences following drug treatment between mouse lines using a two-tailed, paired, Student's $t$ test. The criterion for statistical significance was $P<0.05$.

\section{Acknowledgments}

We wish to thank Sylvia Krüger, Sandra Streu, Maria Jao da Silva, Amela Puskar, Verena Poese (Artemis Pharmaceuticals GmbH), Sarah Waugh, Nerys Aitcheson, Barbara Elcombe, Brian Todd, and Frederick Simeons (CXR Biosciences) for technical advice. This work was supported by ITI Life Sciences, Scotland.

Received for publication February 28, 2008, and accepted in revised form June 11, 2008.

Address correspondence to: Nico Scheer, TaconicArtemis, Neurather Ring 1, 51063 Cologne, Germany. Phone: 49-221-9645343; Fax: 49-221-9645321; E-mail: nico.scheer@taconicartemis.com.
1. Wolf, C.R., Seilman, S., Oesch, F., Mayer, R.T., and Burke, M.D. 1986. Multiple forms of cytochrome P450 related to forms induced marginally by phenobarbital. Differences in structure and in the metabolism of alkoxyresorufins. Biochem. J. 240:27-33.

2. Gonzalez, F.J., and Gelboin, H.V. 1994. Role of human cytochromes P450 in the metabolic activation of chemical carcinogens and toxins. Drug Metab. Rev. 26:165-183.

3. Bergheim, I., Bode, C., and Parlesak, A. 2005. Distribution of cytochrome P450 2C, 2E1, 3A4, and 3A5 in human colon mucosa. BMC Clin. Pharmacol. 5:4.

4. Sarikaya, D., Bilgen, C., Kamataki, T., and Topcu, Z. 2006. Comparative cytochrome P450 -1A1, -2A6, $-2 \mathrm{~B} 6,-2 \mathrm{C},-2 \mathrm{D} 6,-2 \mathrm{E} 1,-3 \mathrm{~A} 5$ and -4B1 expressions in human larynx tissue analysed at mRNA level. Biopharm. Drug Dispos. 27:353-359.
5. Xie, W., et al. 2004. Orphan nuclear receptor-mediated xenobiotic regulation in drug metabolism. Drug Discov. Today. 9:442-449.

6. Stanley, L.A., Horsburgh, B.C., Ross, J., Scheer, N., and Wolf, C.R. 2006. PXR and CAR: nuclear receptors which play a pivotal role in drug disposition and chemical toxicity. Drug Metab. Rev. 38:515-597.

7. Mangelsdorf, D.J., and Evans, R.M. 1995. The RXR heterodimers and orphan receptors. Cell. 83:841-850.

8. Giguere, V. 1999. Orphan nuclear receptors: from gene to function. Endocr. Rev. 20:689-725.

9. Zelko, I., Sueyoshi, T., Kawamoto, T., Moore, R., and Negishi, M. 2001. The peptide near the C terminus regulates receptor CAR nuclear translocation induced by xenochemicals in mouse liver. Mol.
Cell. Biol. 21:2838-2846.

10. Squires, E.J., Sueyoshi, T., and Negishi, M. 2004. Cytoplasmic localization of pregnane $\mathrm{X}$ receptor and ligand-dependent nuclear translocation in mouse liver. J. Biol. Chem. 279:49307-49314.

11. Kliewer, S.A., Goodwin, B., and Willson, T.M. 2002. The nuclear pregnane $X$ receptor: a key regulator of xenobiotic metabolism. Endocr. Rev. 23:687-702.

12. Xie, W., et al. 2000. Humanized xenobiotic response in mice expressing nuclear receptor SXR. Nature. 406:435-439.

13. Maglich, J.M., et al. 2003. Identification of a novel human constitutive androstane receptor (CAR) agonist and its use in the identification of CAR target genes. J. Biol. Chem. 278:17277-17283.

14. Moore, L.B., et al. 2000. Orphan nuclear receptors constitutive androstane receptor and pregnane 
$\mathrm{X}$ receptor share xenobiotic and steroid ligands. J. Biol. Chem. 275:15122-15127.

15. Tzameli, I., Pissios, P., Schuetz, E.G., and Moore, D.D. 2000. The xenobiotic compound 1,4-bis[2(3,5-dichloropyridyloxy)]benzene is an agonist ligand for the nuclear receptor CAR. Mol. Cell. Biol. 20:2951-2958.

16. Lehmann, J.M., et al. 1998. The human orphan nuclear receptor PXR is activated by compounds that regulate CYP3A4 gene expression and cause drug interactions. J. Clin. Invest. 102:1016-1023.

17. Zhang, J., Huang, W., Chua, S.S., Wei, P., and Moore, D.D. 2002. Modulation of acetaminophen-induced hepatotoxicity by the xenobiotic receptor CAR. Science. 298:422-424.

18. Zhou, J., et al. 2006. A novel pregnane X receptormediated and sterol regulatory element-binding protein-independent lipogenic pathway. J. Biol. Chem. 281:15013-15020.

19. Fukuen, S., et al. 2002. Identification of the novel splicing variants for the hPXR in human livers. Biochem. Biophys. Res. Commun. 298:433-438.

20. Savkur, R.S., et al. 2003. Alternative splicing within the ligand binding domain of the human constitutive androstane receptor. Mol. Genet. Metab. 80:216-226.

21. Auerbach, S.S., et al. 2003. Alternatively spliced isoforms of the human constitutive androstane receptor. Nucleic Acids Res. 31:3194-3207.

22. Arnold, K.A., Eichelbaum, M., and Burk, O. 2004. Alternative splicing affects the function and tissue-specific expression of the human constitutive androstane receptor. Nucl. Recept. 2:1.

23. Gardner-Stephen, D., et al. 2004. Human PXR variants and their differential effects on the regulation of human UDP-glucuronosyltransferase gene expression. Drug Metab. Dispos. 32:340-347.

24. Ma, X., et al. 2007. The PREgnane X receptor genehumanized mouse: a model for investigating drugdrug interactions mediated by cytochromes $\mathrm{P} 450$ 3A. Drug Metab. Dispos. 35:194-200.

25. Dotzlaw, H., Leygue, E., Watson, P., and Murphy, L.C. 1999. The human orphan receptor PXR messenger RNA is expressed in both normal and neoplastic breast tissue. Clin. Cancer Res. 5:2103-2107.

26. Hustert, E., et al. 2001. Natural protein variants of pregnane $\mathrm{X}$ receptor with altered transactivation activity toward CYP3A4. Drug Metab. Dispos. 29:1454-1459.
27. Khan, K.K., and Halpert, J.R. 2002. 7-Benzyloxyquinoline oxidation by P450eryF A245T: finding of a new fluorescent substrate probe. Chem. Res. Toxicol. 15:806-814.

28. Kumar, S., Chen, C.S., Waxman, D.J., and Halpert, J.R. 2005. Directed evolution of mammalian cytochrome P450 2B1: mutations outside of the active site enhance the metabolism of several substrates, including the anticancer prodrugs cyclophosphamide and ifosfamide. J. Biol. Chem. 280:19569-19575.

29. Jones, S.A., et al. 2000. The pregnane X receptor: a promiscuous xenobiotic receptor that has diverged during evolution. Mol. Endocrinol. 14:27-39.

30. Meehan, R.R., et al. 1988. Regulation of phenobarbital-inducible cytochrome P-450s in rat and mouse liver following dexamethasone administration and hypophysectomy. Biochem. J. 254:789-797.

31. Sueyoshi, T., Kawamoto, T., Zelko, I., Honkakoski, P., and Negishi, M. 1999. The repressed nuclear receptor CAR responds to phenobarbital in activating the human CYP2B6 gene. J. Biol. Chem. 274:6043-6046.

32. Staudinger, J.L., et al. 2001. The nuclear receptor PXR is a lithocholic acid sensor that protects against liver toxicity. Proc. Natl. Acad. Sci. U. S. A. 98:3369-3374

33. Lukas, G., Brindle, S.D., and Greengard, P. 1971. The route of absorption of intraperitoneally administered compounds. J. Pharmacol. Exp. Ther. 178:562-564.

34. Gibaldi, M., and Perrier, D. 1982. Pharmacokinetics. 2nd edition. Marcel Dekker Inc. New York, New York, USA/Basel, Switzerland. 494 pp.

35. Baes, M., et al. 1994. A new orphan member of the nuclear hormone receptor superfamily that interacts with a subset of retinoic acid response elements. Mol. Cell. Biol. 14:1544-1552.

36. Blumberg, B., et al. 1998. SXR, a novel steroid and xenobiotic-sensing nuclear receptor. Genes Dev. 12:3195-3205.

37. Bertilsson, G., et al. 1998. Identification of a human nuclear receptor defines a new signaling pathway for CYP3A induction. Proc. Natl. Acad. Sci. U. S. A. 95:12208-12213

38. Poland, A., Mak, I., and Glover, E. 1981. Species differences in responsiveness to 1,4 -bis [2-(3,5dichloropyridyloxy)]-benzene, a potent phenobarbital-like inducer of microsomal monooxygenase activity. Mol. Pharmacol. 20:442-450.
39. Wrighton, S.A., et al. 1985. Demonstration in multiple species of inducible hepatic cytochromes P-450 and their mRNAs related to the glucocorticoid-inducible cytochrome $\mathrm{P}-450$ of the rat. $\mathrm{Mol}$. Pharmacol. 28:312-321.

40. Smith, G., et al. 1993. 1,4-Bis[2-(3,5-dichloropyrid yloxy)]benzene, an extremely potent modulator of mouse hepatic cytochrome P-450 gene expression. Biochem. J. 289:807-813.

41. Kocarek, T.A., Schuetz, E.G., Strom, S.C., Fisher, R.A., and Guzelian, P.S. 1995. Comparative analysis of cytochrome P4503A induction in primary cultures of rat, rabbit, and human hepatocytes. Drug Metab. Dispos. 23:415-421.

42. Ma, X., et al. 2007. Rifaximin is a gut-specific human pregnane $\mathrm{x}$ receptor activator. J. Pharmacol. Exp. Ther. 322:391-398.

43. Bauer, B., Hartz, A.M., Fricker, G., and Miller, D.S. 2004. Pregnane X receptor up-regulation of P-glycoprotein expression and transport function at the blood-brain barrier. Mol. Pharmacol. 66:413-419.

44. Schinkel, A.H., Wagenaar, E., van Deemter, L., Mol, C.A., and Borst, P. 1995. Absence of the mdr1a PGlycoprotein in mice affects tissue distribution and pharmacokinetics of dexamethasone, digoxin, and cyclosporin A. J. Clin. Invest. 96:1698-1705.

45. Auerbach, S.S., Dekeyser, J.G., Stoner, M.A., and Omiecinski, C.J. 2007. CAR2 displays unique ligand binding and RXRalpha heterodimerization characteristics. Drug Metab. Dispos. 35:428-439.

46. Zhang, Y., Buchholz, F., Muyrers, J.P., and Stewart, A.F. 1998. A new logic for DNA engineering using recombination in Escherichia coli. Nat. Genet. 20:123-128.

47. Groth, A.C., Olivares, E.C., Thyagarajan, B., and Calos, M.P. 2000. A phage integrase directs efficient site-specific integration in human cells. Proc. Natl. Acad. Sci. U. S. A. 97:5995-6000.

48. Hogan, B.L.M., Beddington, R.S.P., Costantini, F. and Lacy, E. 1994. In Manipulating the mouse embryo: a laboratory manual. Cold Spring Harbor Laboratory Press. Plainview, New York, USA. 253-289.

49. Forrester, L.M., et al. 1992. Relative expression of cytochrome P450 isoenzymes in human liver and association with the metabolism of drugs and xenobiotics. Biochem. J. 281:359-368.

50. Lowry, O.H., Rosebrough, N.Y., Farr, A.L., and Randall, R.J. 1951. Protein measurement with the Folin phenol reagent. J. Biol. Chem. 193:265-275. 